\title{
Effects of taichi on grade 1 hypertension: A study protocol for a randomized controlled trial
}

Sang-Hyun Lee ${ }^{1}$, Byung-Jun Kim², In-Hwa Park' , Eui-Hyoung Hwang ${ }^{1,3}$, Eun Ju Park ${ }^{4}$ Insoo Jang ${ }^{5^{*}}$ and Man-Suk Hwang ${ }^{1,3^{*}}$ (D)

\begin{abstract}
Background: Medication is generally recommended to reduce the morbidity and mortality caused by cardiovascular disease in hypertensive patients. However, considering the difficulties and economic factors associated with long-term medication, interest in taichi as an exercise treatment method has increased recently in Korean medical practice. Numerous studies have suggested that taichi can be used to treat various diseases and that is can affect psychosomatic factors such as anxiety. This study aims to evaluate the effect of taichi in reducing blood pressure among grade 1 hypertensive patients.

Methods/design: In this randomized, active-controlled, assessor-blinded, two parallel-armed trial, 80 grade 1 hypertension patients will be recruited and randomly assigned to the usual care group or to the taichi group ( $n=40$ in each group). Subjects who voluntarily sign a study agreement will be educated in managing their own blood pressure by restricting salt intake, losing weight, moderating alcohol consumption, performing exercise, and regulating dietary intake at their first visit. In addition to self-management, the taichi group will perform two 60-min taichi sessions per week for a total of 8 weeks. Blood pressure will be measured as the primary outcome. In addition, body composition, heart rate, and the perceived intensity and difficulty of the exercise will be measured as secondary outcomes.
\end{abstract}

Discussion: This study is a randomized controlled trial of taichi, which is not widely practiced in Korea. It may provide valuable data on the effects of taichi on hypertension, which will inform non-pharmaceutical treatment options for this disorder.

Trial registration: Clinical Research Information Service, KCT0003632. Registered on 18 March 2019.

Keywords: Hypertension, Taichi, Taichichuan, Taijiquan, Martial arts, Blood pressure

\section{Background}

According to the Korea National Health and Nutrition Examination Survey (2015), the prevalence of hypertension is $27.9 \%$ in Korea, and $36.8 \%$ of Korean adults belong to the pre-hypertension category [1]. The prevalence of age-related hypertension was $52.1 \%$ in males and $51.5 \%$ in

\footnotetext{
* Correspondence: mackayj@naver.com; hwangmansuk@pusan.ac.kr ${ }^{5}$ Department of Internal Medicine, College of Korean Medicine, Woosuk University, 443, Samnye-ro, Samynye-eup, Wanju-Gun, Jeonbuk, Republic of Korea

${ }^{1}$ Department of Rehabilitation Medicine of Korean Medicine, Spine and Joint Center, Pusan National University Korean Medicine Hospital, 20, Geumo-ro, Mulgeum-eup, Yangsan-si, Gyeongnam 50612, Republic of Korea Full list of author information is available at the end of the article
}

females aged $60-69$ years, and $61.7 \%$ in males and $71.3 \%$ in females aged 70 years or older [1]. The higher prevalence in older patients is because age-related reductions in blood vessel elasticity increase the systolic blood pressure (SBP) and decrease the diastolic blood pressure (DBP) [2]. If blood pressure is not controlled, blood vessel damage will occur and lead to the complications of hypertension [2]. If hypertension is not detected early and treated properly, it can lead to complications such as myocardial infarction, stroke, and kidney failure [3].

The goal of controlling hypertension is to reduce the morbidity and mortality caused by cardiovascular disease by maintaining normal blood pressure $[4,5]$. Medication for hypertension is generally recommended for SBP 
greater than $140 \mathrm{mmHg}$ in patients younger than 60 years of age or DBP greater than $90 \mathrm{mmHg}$ regardless of age [6]. However, considering the side effects and economic factors associated with long-term medication, non-medication approaches, such as eating healthily, exercising, ceasing smoking, and moderating alcohol consumption, are widely used to treat and manage hypertension along with pharmacotherapy [7]. In particular, Parker et al. [8] reported that physical activity, including moderate-intensity exercises such as walking or home exercise, reduced the blood pressure in incident hypertension after 15 years of follow-up.

In recent years, there has been increasing interest in taichi as an exercise treatment method for various diseases [9-15]. According to a study conducted by Kim [16], taichi may lower sympathetic tone and increase parasympathetic tone, which may result in changes in the autonomic nervous system. In addition, a randomized controlled study conducted by Tsai et al. [17] suggested that a 12-week period of taichi exercise reduces blood pressure, as well as lipid levels, and improves patient anxiety.

In this study, we analyze the blood pressure, heart rate, and body composition of grade 1 hypertension patients who have been diagnosed during a medical examination in a hospital or had an SBP of 140-159 $\mathrm{mmHg}$ or DBP of $90-99 \mathrm{mmHg}$ in a screening test. We will evaluate the effectiveness of taichi by comparing the control of hypertension in a group practicing usual care with that in an experimental group practicing usual care plus taichi. The effectiveness of taichi for hypertension will, thus, be assessed in this randomized, active-controlled, assessor-blinded, two parallel-armed trial.

\section{Methods/design}

\section{Study design}

The proposed study is a randomized, active-controlled, assessor-blinded, two parallel-armed trial. It will be conducted at Pusan National University Korean Medicine Hospital (PNUKH) in Yangsan, Korea. The study protocol was approved by the institutional review board (IRB) of PNUKH on 16 January 2019 (approval number 2018014) and was registered in the Clinical Research Information Service on 18 March 2019 (KCT0003632). Table 1 is the schedule of enrollment, intervention, and assessments. Table 2 lists all relevant items in the World Health Organization's trial registration data set.

\section{Participants}

Inclusion criteria

To be included, patients must meet all the inclusion criteria:

- They must have been diagnosed with grade 1 hypertension during a health checkup or at the hospital, have SBP of 140 to $159 \mathrm{mmHg}$, or have DBP of 90 to $99 \mathrm{mmHg}$.

- They must understand the study procedures and should be able to follow the advice given

- They must sign the study agreement and voluntarily agree to participate in the study

\section{Exclusion criteria}

People who meet any of the exclusion cannot participate in the trial, that is

- If they have participated in another trial within a month before this study.

Table 1 Schedule of enrollment, intervention, and assessments

\begin{tabular}{|c|c|c|c|c|c|c|c|c|c|c|}
\hline \multirow[t]{2}{*}{ Measure } & \multirow{2}{*}{$\begin{array}{l}\text { Screening } \\
\text { (week 0) }\end{array}$} & \multicolumn{8}{|c|}{ Active treatment } & \multirow{2}{*}{$\begin{array}{l}\text { Follow-up } \\
(12 \text { weeks after } \\
\text { screening })^{\dagger}\end{array}$} \\
\hline & & Week 1 & Week 2 & Week 3 & $\begin{array}{l}\text { Week } 4 \\
(\text { visit 1) }\end{array}$ & Week 5 & Week 6 & Week 7 & $\begin{array}{l}\text { Week } 8 \\
(\text { visit 2) }\end{array}$ & \\
\hline Study agreement & $x$ & & & & & & & & & \\
\hline $\begin{array}{l}\text { Check for participation in other } \\
\text { clinical trials }\end{array}$ & $x$ & & & & & & & & & \\
\hline Sociodemographic characteristics ${ }^{1}$ & $x$ & & & & & & & & & \\
\hline Taichi exercises & & \multicolumn{9}{|c|}{ Two sessions per week } \\
\hline Body composition test & $x$ & & & & & & & & $x$ & $x$ \\
\hline Measurement of vital signs ${ }^{2}$ & $x$ & & & & $x$ & & & & $x$ & $x$ \\
\hline Intensity and difficulty of exercises & & & & & & & & & $x$ & \\
\hline $\begin{array}{l}\text { Number of taichi sessions } \\
\text { attended }\end{array}$ & & & & & & & & & & $x$ \\
\hline Adverse events & & & & & $x$ & & & & $x$ & $x$ \\
\hline
\end{tabular}


Table 2 All relevant items in the World Health Organization's trial registration data set

\begin{tabular}{|c|c|}
\hline Data category & Information \\
\hline $\begin{array}{l}\text { Primary registry and trial } \\
\text { identifying number }\end{array}$ & $\begin{array}{l}\text { Clinical Research Information Service, } \\
\text { KCT0003632 }\end{array}$ \\
\hline $\begin{array}{l}\text { Date of registration in } \\
\text { primary registry }\end{array}$ & 18 March 2019 \\
\hline $\begin{array}{l}\text { Secondary identifying } \\
\text { numbers }\end{array}$ & Not applicable \\
\hline $\begin{array}{l}\text { Source(s) of monetary } \\
\text { or material support }\end{array}$ & $\begin{array}{l}\text { Traditional Korea Medicine R\&D program of } \\
\text { Korea Health Industry Development Institute }\end{array}$ \\
\hline Primary sponsor & Woosuk University \\
\hline Secondary sponsor(s) & Not applicable \\
\hline $\begin{array}{l}\text { Contact for public } \\
\text { queries }\end{array}$ & $\begin{array}{l}\text { Man-Suk Hwang, + 82-55-360-5970, } \\
\text { hwangmansuk@pusan.ac.kr }\end{array}$ \\
\hline $\begin{array}{l}\text { Contact for scientific } \\
\text { queries }\end{array}$ & $\begin{array}{l}\text { Man-Suk Hwang, Department of } \\
\text { Rehabilitation Medicine of Korean Medicine, } \\
\text { Spine and Joint Center, Pusan National } \\
\text { University Korean Medicine Hospital, 20, } \\
\text { Geumo-ro, Mulgeum-eup, Yangsan-si, } \\
\text { Gyeongnam 50612, Republic of } \\
\text { Korea. }\end{array}$ \\
\hline Public title & $\begin{array}{l}\text { Effects of taichi on grade } 1 \\
\text { hypertension: A study protocol for a } \\
\text { randomized controlled trial }\end{array}$ \\
\hline Scientific title & $\begin{array}{l}\text { The Effects of Taichi on Grade } 1 \\
\text { Hypertension: Randomized controlled trial }\end{array}$ \\
\hline $\begin{array}{l}\text { Countries of } \\
\text { recruitment }\end{array}$ & Republic of Korea \\
\hline $\begin{array}{l}\text { Health condition(s) or } \\
\text { problem(s) studied }\end{array}$ & Hypertension \\
\hline Intervention(s) & Taichi \\
\hline $\begin{array}{l}\text { Key inclusion and } \\
\text { exclusion criteria }\end{array}$ & $\begin{array}{l}\text { Inclusion Criteria } \\
\text { o Patients must have been diagnosed } \\
\text { with grade } 1 \text { hypertension during a health } \\
\text { checkup or at the hospital, have SBP of } 140 \\
\text { to } 159 \text { mmHg, or have DBP of } 90 \text { to } 99 \text { mmHg. } \\
\text { o Patients who understand the study } \\
\text { procedures and are able to follow the advice } \\
\text { given } \\
\text { o Patients must sign the study agreement } \\
\text { and voluntarily agree to participate in the } \\
\text { study } \\
\text { Exclusion Criteria } \\
\text { o Patients who have participated in another } \\
\text { trial within a month before this study. } \\
\text { o Patients whose high blood pressure is } \\
\text { deemed by a doctor as too difficult to treat } \\
\text { with exercise because of conditions such } \\
\text { as severe pain or joint deformation. } \\
\text { o Patients who are unable to communicate } \\
\text { properly, for example due to dementia or } \\
\text { mild cognitive impairment. } \\
\text { o Patients who are pregnant. } \\
\text { o Patients who should not be included in } \\
\text { this study based on the investigator's } \\
\text { judgment. }\end{array}$ \\
\hline Study type & $\begin{array}{l}\text { Type of Study: Interventional } \\
\text { Method of allocation: Randomized } \\
\text { Masking: Outcome assessor blinding } \\
\text { Assignment: Two-parallel armed, active } \\
\text { controlled }\end{array}$ \\
\hline
\end{tabular}

Table 2 All relevant items in the World Health Organization's trial registration data set (Continued)

\begin{tabular}{|c|c|}
\hline Data category & Information \\
\hline Date of first enrollment & 17 April 2019 \\
\hline Target sample size & $\begin{array}{l}\text { 1. Number of patients that the trial plans } \\
\text { to enroll in total: } 80 \\
\text { 2. Number of patients that the trial has } \\
\text { enrolled: } 19\end{array}$ \\
\hline Recruitment status & Recruiting \\
\hline Primary outcome(s) & $\begin{array}{l}\text { Blood pressure, which will be measured } \\
\text { at baseline, prior to each hospital visit, } \\
\text { and during the follow-up visit }\end{array}$ \\
\hline $\begin{array}{l}\text { Key secondary } \\
\text { outcomes }\end{array}$ & $\begin{array}{l}\text { Body composition, which will be measured } \\
\text { at baseline, week } 8 \text {, and at the follow-up } \\
\text { visit } \\
\text { Heart rate, which will be measured at } \\
\text { baseline, prior to each visit, and during } \\
\text { the follow-up visit } \\
\text { Intensity and difficulty of the exercises, } \\
\text { which will be assessed once, at the end } \\
\text { of the exercise training. }\end{array}$ \\
\hline Ethics Review & $\begin{array}{l}\text { 1. Status: Approved } \\
\text { 2. Date of approval: } 16 \text { January } 2019 \\
\text { 3. Name and contact details of Ethics } \\
\text { committee: Pusan National University } \\
\text { Korean Medicine Hospital IRB (approval } \\
\text { number 2018014), 20, Geumo-ro, } \\
\text { Mulgeum-eup, Yangsan-si, } \\
\text { Gyeongnam 50612, Republic } \\
\text { of Korea, + 82-55-360-5902 }\end{array}$ \\
\hline Completion date & 15 January 2020 \\
\hline Summary results & Not applicable: protocol \\
\hline $\begin{array}{l}\text { Individual patient data } \\
\text { sharing statement }\end{array}$ & $\begin{array}{l}\text { 1. Plan to share individual patient data: } \\
\text { Decided } \\
\text { 2. Plan description: The datasets used or } \\
\text { analyzed during the study can be requested } \\
\text { from the corresponding author }\end{array}$ \\
\hline
\end{tabular}

- If their high blood pressure is deemed by a doctor as too difficult to treat with exercise because of conditions such as severe pain or joint deformation.

- If they are unable to communicate properly, for example due to dementia or mild cognitive impairment. - If they are pregnant.

- If they should not be included in this study based on the investigator's judgment.

\section{Discontinuation and dropout criteria}

Participants will be withdrawn from the study:

- If they are found to have a previously undiagnosed severe disease after enrollment and before the start of the clinical trial.

- If they have another disease (except hypertension) that may influence the results of the trial. - If they miss two consecutive assessment visits.

- If they ask to be withdrawn from the trial.

- If they are lost to follow-up. 


\section{Recruitment}

Patients will be recruited using advertisements on the bulletin boards of Pusan National University Yangsan Hospital (PNUYH) and PNUKH, at local public health centers, and at other local government offices. Patients who are interested in participating will first be screened to determine their eligibility. Eligible patients will receive an explanation of the study and can voluntarily decide whether they wish to participate. If the patient consents to the study terms, a clinical research coordinator (CRC) will check whether they are suitable in accordance with the inclusion and exclusion criteria. Then, the patient will be assigned randomly to the taichi group or the usual care group. After allocation, the CRC will schedule their treatment procedure. The first participant was enrolled on 17 April 2019.

\section{Randomization}

A statistician who is not involved in conducting or assessing the clinical trial will generate a randomization sequence using the statistical program SAS ${ }^{\circ}$, Version 9.4 (SAS institute. Inc., Cary, NC). Using the sequence, the statistician will prepare 80 sealed envelopes, 40 of which will contain an A and 40 a B based on blocked randomization.
Once a participant has signed the informed consent form, the CRC will open the next envelope in the sequence and inform the practitioner of the participant's assignment to the experimental group or the usual care group (Fig. 1).

\section{Blinding}

Since blinding of the intervention is impossible, the group allocation will not be concealed and the participants will not be blind. Instead, the outcome assessor and data analyst will be blind to the allocation and they will not participate in recruitment or the intervention. The outcome assessor will assess participants at the study visits. The data analyst will analyze the statistical data to prevent selective reporting of outcome variables. Unblinding will be permitted only when it is necessary to reveal the participant's allocated intervention, such as in cases of severe side effects, at the discretion of the assessor.

\section{Education levels of the practitioners}

All the physicians participating in this study as practitioners or researchers are licensed by the Ministry of Health and Welfare of Korea and have at least one year of clinical experience in Korean medicine. These practitioners will be

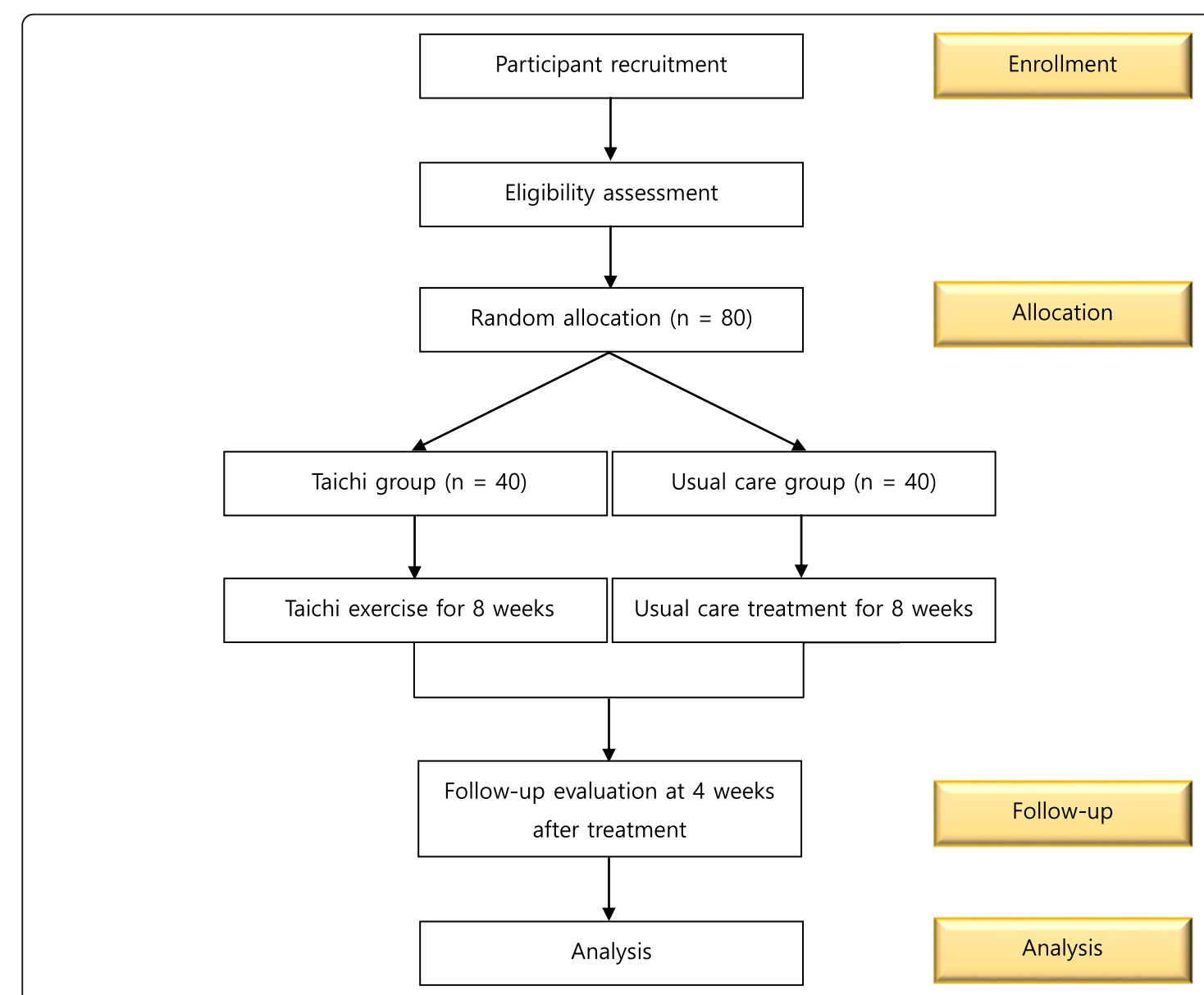

Fig. 1 Flowchart outlining the study timeline, including enrollment, allocation, follow-up, and analysis 
adequately trained so that they closely adhere to the research protocols and are familiar with the research treatment methods. All the taichi educators have been trained as Korean rehabilitation medical trainees or specialists in taichi exercise and have at least one year of taichi experience. Among them, the research director of this study has more than ten years of taichi experience.

\section{Intervention}

At their first visit, patients in both groups will be educated in managing their own blood pressure by restricting salt intake, losing weight, moderating alcohol consumption, exercising, and regulating their diet. Additionally, patients in both groups will be asked not to do any intense exercise that could influence the results of the trial. After this education, the usual care group will self-manage their own blood pressure for 8 weeks.

In addition to self-management, the experimental group will perform taichi as the study intervention. The taichi used in this study is Chen-style 18-form taichi, which will be conducted over two 60-min sessions per week for a total of 8 weeks ( $80 \%$ compliance and more than 13 sessions in total). In each session, patients will perform $10 \mathrm{~min}$ of warm-up exercises, $40 \mathrm{~min}$ of taichi, and $10 \mathrm{~min}$ of cool-down exercises. To maximize adherence to the study protocol, the intervention will mostly be performed in the 6th floor ward at PNUKH. If group sessions are needed, the practitioners can visit external locations. The patients in the experimental group will receive a reference book on taichi that will promote their retention of the exercises. A daily log will record how many exercises each patient has conducted. At weeks 4 and 8 , both groups will visit the hospital for an assessment. After treatment, both groups will be monitored for an additional 4 weeks of follow-up.

\section{Outcome assessment}

At the initial screening visit, the CRC will explain the study protocol to the patient. Then, the participants will be asked about their sociodemographic characteristics, including age, gender, occupation, past history, present illness, and medications, at an isolated room for allocation concealment. All adverse events will be recorded, and the practitioners will check the severity of the events and decide the continuance of the trial. Follow-up assessments will be performed once at 12 weeks after the initial screening visit (Table 1, Fig. 1).

\section{Primary outcome measurements}

Blood pressure will be the primary outcome of this trial. The CRC will assess the participant's blood pressure in a stable state using an automatic electronic blood pressure monitor (HBP-1300, Omron Dalian Co., Ltd., China). To obtain accurate data, the measurements will be conducted three times and their mean value will be used as the outcome. Blood pressure will be measured at baseline (assessment 1), prior to each of the visits (assessments 2 and 3), and during the follow-up visit (assessment 4). The primary endpoint is week 8 (assessment 3).

\section{Secondary outcome measurements}

Body composition is one of the secondary outcomes of this trial. The body composition test assesses each participant's weight, body fat mass, body mass index, percentage of body fat, and weight-to-hip ratio. The participant will stand barefoot on the Inbody body composition analyzer (Inbody 770; Inbody Co., Ltd., South Korea). After their weight has been measured, the participant will grasp the handles and the machine will pass multifrequency signals through their body to obtain the impedance corresponding to each frequency. Using these measured impedance values, the machine will calculate how much body fat they have. The test will be conducted at baseline (assessment 1 ), week 8 (assessment 3), and at the follow-up visit (assessment 4).

Heart rate is another secondary outcome of this trial. The heart rate of participants will assessed when they are in a stable state at the same time as their blood pressure is measured. Thus, measurements will be conducted at baseline (assessment 1), prior to each of the visits (assessments 2 and 3), and during the follow-up visit (assessment 4).

The intensity and difficulty of the exercises are also secondary outcomes. The aim is to compare their subjective assessment of the intensity of the exercise with its absolute intensity. The experimental group will be asked four questions.

1. Was this exercise routine easy to follow?

2. Is taichi useful for improving your health?

3. Was the taichi conducted at appropriate time for you?

4. Is the reference book helpful for performing taichi on your own?

Participants will answer each question using a 0 to 10 category scale.

They will rate the difficulty of the taichi exercises using a 0 to 10 visual analog scale, on which 0 indicates no difficulty and 10 indicates the maximum possible difficulty the person can imagine. Both surveys will be conducted once at the end of the final taichi session. Thus, the measurement point will be week 8 (assessment 3 ).

\section{Sample size}

This study took into account the results of a previous study [18] that used taichi as the main evaluation index. The calculated sample size necessary for the $t$-test was 36 subjects in each group, which was conducted with a 
G power analysis with an effect size of 0.67 , test power of 0.80 , and significance level of 0.05 . Considering a dropout rate of $10 \%$, we aim to recruit a total of 40 subjects in each group.

\section{Statistical analysis plan}

Continuous variables will be expressed as mean \pm standard deviation, and categorical variables will be expressed as $n$ (\%). The demographic baseline information (age, gender, occupation, past history, present illnesses, and medications) will be tested using the chi-squared test and independent $t$-test.

As the primary statistical analysis, the effectiveness of taichi will be tested by calculating the differences in the maximum SBP and minimum DBP before (baseline) and after (week 8) treatment for each test subject. Comparisons of the within-group differences will be performed using a paired $t$-test and between-group differences using an independent $t$-test. If the data do not have a normal distribution, they will be tested using a nonparametric test (a Wilcoxon signed-rank test or Wilcoxon rank-sum test).

The heart rate and the body composition of the participants will be tested to verify the effectiveness of taichi by calculating the difference between before (baseline) and after (week 8) the treatment as described above.

The results of the questions regarding the intensity and difficulty of the exercises will be evaluated by the researchers, and the numerical value will be analyzed by a simple descriptive statistical method. We will perform a simple correlation analysis or simple regression analysis to determine whether there is a correlation between the differences in blood pressure and the intensity and difficulty of the exercise as perceived by the experimental group.

Information regarding adverse events will be collected through patient reports and researchers' observations. The frequency of adverse events will be analyzed by a chi-squared test or Fisher's exact test.

All statistical analyses will be conducted in a two-sided manner, with a significance level of 5\%. In addition, we will use an intention-to-treat analysis for missing data in the primary and secondary analyses. The last-observationcarried-forward and multiple imputation, which are widely used in clinical research, will be applied to missing data and additional multiple imputation or regression analysis will be used to check any differences.

\section{Safety}

Because the intervention is a simple exercise rather than untested drugs or medical devices, we do not expect any adverse events will be caused by the general Korean medical treatment. However, the investigators will inform the participants of all possible adverse events that may occur after taichi and instruct them to report any such adverse events. All adverse events recorded during the research period will be analyzed and reported. In general, due to the interventional characteristics of exercise, simple muscular pain may occur. We will provide beverages such as bottled water or green tea during the exercise sessions for the participants. To prevent falls and severe muscle aches, a chair will be available during exercise sessions. There will also be a space where participants can sit and relax. If a direct injury occurs in connection with this study, appropriate medical action may be taken, as determined by the investigator.

A data monitoring committee (DMC) comprising members of staff from the clinical research service institution (Woosuk University) will periodically monitor the study by telephone, e-mail, and visits, if necessary. The DMC will be composed of one statistician and one specialist in clinical research methodology. They will review the progress of the study and check all case report forms. In addition, they will check whether the study has followed the study protocol. If a problem is identified, the DMC will modify the protocol if necessary. If modifications to the protocol are required during the study, the modified protocol will be submitted by the clinical trial review committee to the IRB for approval. However, neither auditing nor an interim analysis are planned during the trial.

Additionally, as we will not collect any biological samples and do not intend to use the participants' data in future studies, we do not need any additional consent for collection and use of participant data or biological specimens in ancillary studies. In accordance with government regulations and standards, all documents related to the conduct of a clinical trial must be kept by the director of clinical research, or the director of the institution and the clinical research manager, for at least 3 years after the completion of the clinical trial.

\section{Ethics and dissemination}

As this clinical study was prepared with patient rights and well-being in mind based on the Declaration of Helsinki, the clinical researchers will follow the research plan and will actively respond to any problems raised by the participants. Additional file 1 contains a completed SPIRIT checklist.

Before patients are asked to participate in this clinical study, the researchers will explain to them all the details of the research. They must voluntarily agree to participate in the research. The English initials of the names of the participants in the trial will be recorded and identifying information will be managed using a subject identification code list to prevent personal information, such as social security numbers, from being leaked. Other researchers and research organizations will be able to view the clinical research data collected during reviews by the DMC, IRB inspections and assessments, and government 
surveys. On completion of the trial, the researchers will consult with the clinical research service institution (Woosuk University) to prepare a report of this study. The study findings will be disseminated in peer-reviewed journals and presented at national and international conferences. Additionally, we will share deidentified individual patient data. The datasets used or analyzed during the study can be requested from the corresponding author.

\section{Discussion}

When searching for research conducted in Korea into the effects of taichi on blood pressure, we found studies that assessed the effects of taichi on waist circumference and blood pressure of the elderly [18] and the effect of taichi on the cardiac autonomous nervous system and blood pressure of elderly women [16]. These studies on taichi mainly had elderly participants. In addition, in a systematic review by Hwang et al. [19], only randomized controlled trials from China, Italy, United Kingdom, USA, Hong Kong, and Taiwan were analyzed, and no randomized controlled trials from Korea were included.

A major limitation of this study is that the participants cannot be blinded due to the nature of the intervention. Because the experimental group has to perform taichi, the participants will know whether they belong to the experimental group or the comparator group. This knowledge may affect the results of the study by influencing other behaviors, which may, thus, differ between the groups.

A strength of this study is that it will evaluate the effects of taichi in ordinary adults aged 19 to 70 years instead of being limited to the elderly. In addition, this study is a randomized controlled trial of taichi, which is not widely practiced in Korea. Taichi is easy to perform and not strenuous, so it can easily be practiced at home by almost anyone. As a result, this study may provide valuable data on the effects of taichi on hypertension.

\section{Trial status}

The current protocol is version 1.7, dated 28 November 2019. Recruitment began on 17 April 2019. The approximate completion date for recruitment is in April 2020. This trial was prospectively registered before recruitment began.

\section{Supplementary information}

Supplementary information accompanies this paper at https://doi.org/10. 1186/s13063-019-4028-6.

Additional file 1. SPIRIT 2013 Checklist: Recommended items to address in a clinical trial protocol and related documents.

\section{Abbreviations}

CRC: Clinical research coordinator; DBP: Diastolic blood pressure; DMC: Data monitoring committee; IRB: Institutional review board; PNUKH: Pusan
National University Korean Medicine Hospital; PNUYH: Pusan National University Yangsan Hospital; SBP: Systolic blood pressure

\section{Acknowledgements}

The authors express their gratitude to all participants, past, and future, in this clinical trial. In addition, we would like to thank Editage (www.editage.co.kr) for English language editing.

\section{Authors' contributions}

SHL designed the study and drafted the manuscript. ISJ planned the statistical strategy. EJP was actively involved in the sample size calculation and random allocation. EHH is the principal investigator at the hospital and, along with BJK, will teach the patients taichi. As investigators at the hospital, IHP, BJK, and MSH were involved in the study design and IRB approval. ISJ and $\mathrm{MSH}$ are the principal investigators of the overall research project and have the final responsibility for publication. All authors have read, revised, and approved the final version of the manuscript.

\section{Funding}

This study was supported by the Traditional Korean Medicine Research \& Development program funded by the Ministry of Health \& Welfare through the Korea Health Industry Development Institute (HB16C0023). This funding source had no role in the design of the study and will not have any role during collection, management, analysis, interpretation of data, writing of the report, and the decision to submit the report for publication.

\section{Availability of data and materials}

The datasets used or analyzed during the study can be requested from the corresponding author.

\section{Ethics approval and consent to participate}

This study has been approved by the IRB of PNUKH (approval number 2018014). All participants must sign the latest version of the informed consent form approved by the IRB. This trial has been registered at the Clinical Research Information Service (КСT0003632), which is one of the World Health Organization's International Clinical Trials Registry Platforms. If protocol modifications are necessary, they will be reported to the IRB. The personal information of subjects will be kept secret and will be processed anonymously.

\section{Consent for publication}

Not applicable.

\section{Competing interests}

The authors declare that they have no competing interests.

\section{Author details}

${ }^{1}$ Department of Rehabilitation Medicine of Korean Medicine, Spine and Joint Center, Pusan National University Korean Medicine Hospital, 20, Geumo-ro, Mulgeum-eup, Yangsan-si, Gyeongnam 50612, Republic of Korea. ${ }^{2}$ School of Korean Medicine, Pusan National University, Yangsan, Republic of Korea. ${ }^{3}$ Division of Clinical Medicine, School of Korean Medicine, Pusan National University, Yangsan, Republic of Korea. ${ }^{4}$ Family Medicine Clinic, Pusan National University Yangsan Hospital, Yangsan, Republic of Korea. ${ }^{5}$ Department of Internal Medicine, College of Korean Medicine, Woosuk University, 443, Samnye-ro, Samynye-eup, Wanju-Gun, Jeonbuk, Republic of Korea.

Received: 30 August 2019 Accepted: 23 December 2019

Published online: 13 February 2020

\section{References}

1. Ministry of Health \& Welfare DoHP. Korea National Health and Nutrition Examination Survey; 2016. p. 50.

2. Mosley WJ II, Lloyd-Jones DM. Epidemiology of Hypertension in the Elderly. Clin Geriatr Med. 2009:25(2):179-89.

3. Turgut F, Yesil Y, Balogun RA, Abdel-Rahman EM. Hypertension in the elderly: unique challenges and management. Clin Geriatr Med. 2013;29(3): 593-609.

4. Carlberg B, Nilsson PM. Hypertension in the Elderly: What Is the Goal Blood Pressure Target and How Can This Be Attained? Curr Hypertens Rep. 2010;12(5):331-4. 
5. Hartley L, Flowers N, Lee MS, Ernst E, Rees K. Taichi for primary prevention of cardiovascular disease. Cochrane Database Syst Rev. 2014;4:Cd010366.

6. Nilsson PM. Blood pressure strategies and goals in elderly patients with hypertension. Exp Gerontol. 2017;87:151-0.

7. Mahmood S, Shah KU, Khan TM, Nawaz S, Rashid H, Baqar SWA, et al. Nonpharmacological management of hypertension: in the light of current research. Ir J Med Sci. 2019;188(2):437-52.

8. Parker ED, Schmitz KH, Jacobs DR Jr, Dengel DR, Schreiner PJ. Physical activity in young adults and incident hypertension over 15 years of followup: the CARDIA study. Am J Public Health. 2007;97(4):703-9.

9. Ahn Y-J, Jo S-H, Lee S-H, Lim J-H. The review study on Yoga, Qigong, and Taichi interventions for anxiety: Based on Korean journal articles from 2009 to 2015. J Oriental Neuropsych. 2016;27(1):23-31.

10. Ji HQ. The Effect of Taichi Exercise on Attention Concentration and Dynamic Balance in Children with ADHD [master's thesis]. Pusan: Pusan National University; 2015.

11. Kim KL, Bae SJ, Seo HK. The Effect of Taichi on Health Related Fitness and Cognitive Function in Elderly Women with Dementia. J Kor Soc Rhythmic Exercises. 2018;11(2):51-8.

12. Kim Y-S, Park M-S, Kwak Y-S. 12-Week Taichi Exercise on Physical Fitness, Blood Pressure, and Cholesterol in Elderly Women. J Kor Soc Wellness. 2012;7(1):119-28.

13. Lee HY. The Effect of Taichi for Patients with Type 2 Diabetes - Analysis According to the Styles of Taichi Considering the Intensity: Systematic Review [master's thesis]. Pusan: Pusan National University School of Korean Medicine; 2015.

14. Li F, Harmer P, Fitzgerald K, Eckstrom E, Stock R, Galver J, et al. Taichi and postural stability in patients with Parkinson's disease. N Engl J Med. 2012;366(6):511-9.

15. Son JT, Hwang H. Comparison of Subjective Symptoms, Physical Fitness, Depression and Self-efficacy before and after Taichi in Patients with Rheumatoid Arthritis: Based on 3 Years Data. J Muscle Joint Health. 2018:25(3):230-9.

16. Kim J-H. The Effect of Taichi Chuan Training on the Automatic Cardiac Nerve Activity and Blood Pressure in Elderly Women with Hypertension. J Kor Soc Dance Sci. 2014;31(1):157-68.

17. Tsai JC, Wang WH, Chan P, Lin LJ, Wang $C H$, Tomlinson B, et al. The beneficial effects of Taichi Chuan on blood pressure and lipid profile and anxiety status in a randomized controlled trial. J Altern Complement Med. 2003:9(5):747-54.

18. Lee YM. The effects of Taichi on waist circumference and blood pressure in the elderly. J Physical Therapy Sci. 2017;29(1):172-5.

19. Hwang E-H, Heo K-H, Lee G-S. Effect of Taichi as Therapeutic Exercise on Blood Pressure Rapid Systematic Review. J Oriental Rehab Med. 2013;23(1):101-13.

\section{Publisher's Note}

Springer Nature remains neutral with regard to jurisdictional claims in published maps and institutional affiliations.

Ready to submit your research? Choose BMC and benefit from:

- fast, convenient online submission

- thorough peer review by experienced researchers in your field

- rapid publication on acceptance

- support for research data, including large and complex data types

- gold Open Access which fosters wider collaboration and increased citations

- maximum visibility for your research: over $100 \mathrm{M}$ website views per year

At $\mathrm{BMC}$, research is always in progress.

Learn more biomedcentral.com/submissions 